УДК 519.218.2

\title{
On Long-time Behaviors of States of Galton-Watson Branching Processes Allowing Immigration
}

\author{
Azam A. Imomov* \\ State Testing Center under the Cabinet of MRU \\ Karshi State University \\ Kuchabag, 17, Karshi city, 180100
}

Uzbekistan

Received 13.06.2015, received in revised form 01.08.2015, accepted 02.09.2015

We observe the discrete-time Branching Process allowing Immigration. Limit properties of transition functions and their convergence to invariant measures are investigated. In the critical situation a speed of this convergence is defined.

Keywords: branching process, immigration, transition functions, invariant measures, ratio limit property, rate of convergence.

DOI: $10.17516 / 1997-1397-2015-8-4-394-405$

\section{Introduction}

Let the random function $X_{n}$ denote the successive population size of the Galton-Watson Branching Process allowing Immigration (GWPI) at the moment $n \in \mathbb{N}_{0}$, here $\mathbb{N}_{0}=\{0\} \cup\{\mathbb{N}=$ $1,2, \ldots\}$. The state sequence $\left\{X_{n}\right\}$ is a homogeneous Markov chain with state space on $\mathbb{N}_{0}$ and can be expressed recursively as

$$
X_{n}=\sum_{k=1}^{X_{n-1}} \xi_{n k}+\eta_{n}, \quad \text { for } n \in \mathbb{N},
$$

where independent and identically distributed (i.i.d.) random variables $\xi_{n k}$ denote the offspring number of $k$-th individual in the $(n-1)$-th generation, and i.i.d. variables $\eta_{n}$ are not depend on $\xi_{n k}$ interpreted as number of immigrants-individuals at the moment $n$. We assume $X_{0}=0$ and the process starts owing to immigrants. Each individual reproduces independently of each other and according to the offspring law $p_{k}:=\mathbb{P}\left\{\xi_{11}=k\right\}$. With probability $h_{j}:=\mathbb{P}\left\{\eta_{1}=j\right\}$ arrive $j \in \mathbb{N}_{0}$ immigrants in population in each moment $n \in \mathbb{N}$. These individuals undergo further transformation by the reproduction law $\left\{p_{j}\right\}$. Throughout the paper we assume $p_{0}>0$ and $\sum_{j \in \mathbb{N}_{0}} h_{j}=1$.

We denote $\mathcal{S} \subseteq \mathbb{N}_{0}$ to be the state space of the chain $\left\{X_{n}\right\}$. It is indicated by $n$-step transition functions

$$
p_{i j}^{(n)}=\mathbb{P}_{i}\left\{X_{n}=j\right\}:=\mathbb{P}\left\{X_{k+n}=j \mid X_{k}=i\right\},
$$

for any $n, k \in \mathbb{N}_{0}$. Let

$$
\mathcal{P}_{n}^{(i)}(s):=\mathbb{E}_{i} s^{X_{n}}=\sum_{j \in \mathcal{S}} p_{i j}^{(n)} s^{j}
$$

*imomov_azam@mail.ru

(c) Siberian $\bar{F}$ ederal University. All rights reserved 
is probability generating function (PGF). Denoting

$$
G(s):=\sum_{j \in \mathbb{N}_{0}} h_{j} s^{j} \quad \text { and } \quad F(s):=\sum_{j \in \mathbb{N}_{0}} p_{j} s^{j}
$$

one can see

$$
\mathcal{P}_{n+1}^{(i)}(s)=G(s) \cdot \mathcal{P}_{n}^{(i)}(F(s))
$$

From (1.1) we have

$$
\mathcal{P}_{n}^{(i)}(s)=\left[F_{n}(s)\right]^{i} \prod_{k=0}^{n-1} G\left(F_{k}(s)\right),
$$

where $F_{n}(s)$ is $n$-fold iterate of PGF $F(s)$; see, e.g., [1, p. 263]. Now it is clear the probabilities $\left\{p_{i j}^{(n)}\right\}$ are completely defined by means of probabilities $\left\{p_{j}\right\}$ and $\left\{h_{j}\right\}$.

Classification of states of the chain $\left\{X_{n}\right\}$ is one of fundamental problems in theory of GWPI. Direct differentiation of (1.2) gives us

$$
\mathbb{E}_{i} X_{n}=\sum_{j \in \mathcal{S}} j p_{i j}^{(n)}= \begin{cases}\left(\frac{\alpha}{A-1}+i\right) A^{n}-\frac{\alpha}{A-1}, & \text { when } A \neq 1, \\ \alpha n+i, & \text { when } A=1,\end{cases}
$$

where $A=F^{\prime}(1)$ and $\alpha=G^{\prime}(1)$. The received formula for $\mathbb{E}_{i} X_{n}$ shows that classification of states of GWPI depends on the value of parameter $A$ is the mean number of direct descendants of single individual as a result of transformation for one-step generation. Process $\left\{X_{n}\right\}$ is classified as sub-critical, critical and supercritical if $A<1, A=1$ and $A>1$ accordingly.

The above described evolution process of individuals was considered first by Heathcote [3] in 1965. Further long-term properties of states and a problem of existence and uniqueness of invariant measures of GWPI were investigated in papers of Seneta $[8,10,11]$, Pakes [4-7] and by many other authors. Therein some moment conditions for PGF $F(s)$ and $G(s)$ was required to be satisfied. In aforementioned works of Seneta the ergodic properties of $\left\{X_{n}\right\}$ were investigated. He has proved that in cases $A \leqslant 1$ there is a unique invariant measure $\left\{\mu_{k}, k \in \mathcal{S}\right\}$ and besides $\mu_{0}=1$. Heathcote [2] and Pakes [7] have shown that in supercritical case $\mathcal{S}$ is transient. In the critical case if the first moment of immigration law $\alpha:=G^{\prime}(1)$ is finite, then $\mathcal{S}$ can be transient, null-recurrent or ergodic. In this case, if in addition to assume that $2 B:=F^{\prime \prime}(1)<\infty$, properties of $\mathcal{S}$ depend on value of parameter $\lambda=\alpha / B$ : if $\lambda>1$ or $\lambda<1$, then $\mathcal{S}$ is transient or null-recurrent accordingly. In the case when $\lambda=1$, Pakes [6] and Zubkov [15] studied necessary and sufficient conditions for a null-recurrence property. Limiting distribution law for critical process $\left\{X_{n}\right\}$ was found first by Seneta [9]. By them it has been proved under the condition of $0<\lambda<\infty$ the normalized process $X_{n} / n$ has limiting Gamma distribution with density function

$$
\frac{1}{B \Gamma(\lambda)}\left(\frac{x}{B}\right)^{\lambda-1} e^{-x / B}, \text { for } x>0,
$$

where $\Gamma(*)$ is Euler's Gamma function. This result without reference to Seneta has been established also by Pakes [6].

More recent researches on asymptotic properties of process contain in papers [12-14] in which the Bernoulli type GWPI was considered, i.e. both $\xi_{n k}$ and $\eta_{n}$ obey the Binomial distribution law. Clearly Bernoulli type GWPI is a special class in the general theory of Branching Processes. In this paper we consider processes in which both offspring law and immigration law are arbitrary. 
In Section 2 invariant properties of GWPI will be investigated. The analogue of the Ratio Limit Property Theorem for transition functions $\left\{p_{i j}^{(n)}\right\}$ will be proved (Theorem 1 below).

The Section 3 is devoted to estimate of speed of convergence of $\left\{n^{\lambda} p_{i j}^{(n)}\right\}$ to invariant measures in the critical case.

\section{Invariant property of transition functions}

First we are interested in long-time behavior of ratio $p_{i j}^{(n)} / p_{00}^{(n)}$ for any $i, j \in \mathcal{S}$. Having designation $\mathcal{P}_{n}(s):=\mathcal{P}_{n}^{(0)}(s)$, it follows from (1.2) that

$$
\frac{\mathcal{P}_{n}^{(i)}(s)}{\mathcal{P}_{n}(s)} \longrightarrow q^{i}, \quad \text { as } n \rightarrow \infty,
$$

because of $F_{n}(s) \rightarrow q$ for $0 \leqslant s<1$; see [17, p. 53]. Recall that $q$ is an extinction probability of the simple branching process without immigration with $\mathrm{PGF} F(s)$. It is the least nonnegative solution of $q=F(q)$, and that $q=1$ if $A \leqslant 1$ and $q<1$ if $A>1$. Putting $s=0$ in (2.1) implies $p_{i 0}^{(n)} / p_{00}^{(n)} \rightarrow q^{i}$ as $n \rightarrow \infty$. On purpose to receive the statement generally for all $j \in \mathcal{S}$, we write

$$
\mathcal{P}_{n+1}(s)=\mathcal{P}_{n}(s) \cdot G\left(F_{n}(s)\right)
$$

and from here and considering the properties of PGF one can calculate derivatives of $j$-th order:

$$
\frac{\partial^{j} \mathcal{P}_{n+1}(s)}{\partial s^{j}}=\frac{\partial^{j} \mathcal{P}_{n}(s)}{\partial s^{j}} \cdot G\left(F_{n}(s)\right)+D_{j, n}(s),
$$

for all $0 \leqslant s<1$, where expression $D_{j, n}(s)$ is a power series with nonnegative coefficients. Since $p_{0 j}^{(n)}=\partial^{j} \mathcal{P}_{n+1}(s) /\left.\partial s^{j}\right|_{s=0}$, from last received results we obtain

$$
\frac{p_{0 j}^{(n+1)}}{p_{00}^{(n+1)}} \geqslant \frac{p_{0 j}^{(n)}}{p_{00}^{(n)}}
$$

So the sequence of functions $\left\{p_{0 j}^{(n)} / p_{00}^{(n)}\right\}$ monotonously increases as $n \rightarrow \infty$. In our conditions $p_{00}^{(n)}>0$ for any $n \in \mathbb{N}$. Therefore this sequence converges increasing to the finite non-negative limit which we will designate as $v_{j}$ :

$$
\frac{p_{0 j}^{(n)}}{p_{00}^{(n)}} \uparrow v_{j}<\infty, \quad \text { as } n \rightarrow \infty .
$$

Let's consider now more general ratio $p_{i j}^{(n)} / p_{00}^{(n)}$. Denoting

$$
\mathcal{U}_{n}^{(i)}(s):=\sum_{j \in \mathcal{S}} \frac{p_{i j}^{(n)}}{p_{00}^{(n)}} s^{j}, \quad \text { for } \quad 0 \leqslant s<1,
$$

we write the following equalities:

$$
\mathcal{U}_{n}^{(i)}(s)=\sum_{j \in \mathcal{S}} \frac{p_{i j}^{(n)}}{p_{00}^{(n)}} s^{j}=\left[F_{n}(s)\right]^{i} \frac{\mathcal{P}_{n}(s)}{\mathcal{P}_{n}(0)}=\left[F_{n}(s)\right]^{i} \mathcal{U}_{n}(s),
$$


where

$$
\mathcal{U}_{n}(s)=\sum_{j \in \mathcal{S}} \frac{p_{0 j}^{(n)}}{p_{00}^{(n)}} s^{j} .
$$

Now wee prove the following Ratio Limit Property (RLP) Theorem.

Theorem 1. The general GWPI satisfies the $R L P$ for all $i, j \in \mathcal{S}$ :

$$
\lim _{n \rightarrow \infty} \frac{p_{i j}^{(n)}}{p_{00}^{(n)}}=q^{i} v_{j}<\infty .
$$

An appropriate $P G F$ of $v_{j}=\lim _{n \rightarrow \infty} p_{0 j}^{(n)} / p_{00}^{(n)}$ is

$$
\mathcal{U}(s)=\sum_{j \in \mathcal{S}} v_{j} s^{j}
$$

and it satisfies the functional equation

$$
\sigma \cdot \mathcal{U}(s)=G(s) \cdot \mathcal{U}(F(s)),
$$

in a region of its convergence, where $\sigma:=G(q)$.

Proof. The statement (2.4) immediately follows from relations (2.2), (2.3) and that fact $F_{n}(s) \rightarrow q$ uniformly for $0 \leqslant s \leqslant r<1$ as $n \rightarrow \infty$.

To prove the justice of the equation (2.5) we consider together the relations (1.1), (2.3) and the known equality $\mathcal{P}_{n+1}(s)=\mathcal{P}_{n}(s) \cdot G\left(F_{n}(s)\right)$ and receive the following equalities:

$$
\begin{aligned}
\mathcal{U}_{n+1}^{(i)}(s) & =\left[F_{n+1}(s)\right]^{i} \mathcal{U}_{n+1}(s)=\left[F_{n}(F(s))\right]^{i} \frac{\mathcal{P}_{n+1}(s)}{\mathcal{P}_{n+1}(0)}= \\
& =\left[F_{n}(F(s))\right]^{i} \frac{G(s) \cdot \mathcal{P}_{n}(F(s))}{G\left(F_{n}(0)\right) \cdot \mathcal{P}_{n}(0)}=\frac{G(s)}{G\left(F_{n}(0)\right)} \cdot \mathcal{U}_{n}^{(i)}(F(s)) .
\end{aligned}
$$

Taking limit as $n \rightarrow \infty$ from here we get to $(2.5)$.

The Theorem is proved.

PGF $\mathcal{U}(s)$ as a power series represents a continuous function in field of $0 \leqslant s<1$. According to properties of PGF it converges for all $s \in[0 ; 1-\varepsilon]$ and for any arbitrary small constant $\varepsilon>0$.

Repeatedly using the iteration of PGF $F(s)$ in the equation (2.5) leads us to the following relation:

$$
\sigma^{n} \mathcal{U}(s)=\mathcal{P}_{n}(s) \mathcal{U}\left(F_{n}(s)\right)
$$

The transition function analogue of $(2.6)$ is

$$
\sigma^{n} \cdot v_{j}=\sum_{i \in \mathcal{S}} v_{i} p_{i j}^{(n)} .
$$

Equality (2.7) indicates that the set of non-negative numbers $\left\{v_{j}, j \in \mathcal{S}\right\}$ represents an invariant measure for the chain $\left\{X_{n}\right\}$.

Due to the condition $p_{00}^{(n)}>0$ and the equality (2.7), all of $v_{j}<\infty$ and $v_{j}>0$ for $j \in \mathcal{S}$. And $v_{0}=1$ as well. Then by definition of the process $\left\{X_{n}\right\}$ and owing to $(2.7)$ we have the following chain of equalities:

$$
\begin{aligned}
\sigma^{n} & =\sigma^{n} \cdot v_{0}=\sum_{i \in \mathcal{S}} v_{i} p_{i 0}^{(n)}= \\
& =\sum_{i \in \mathcal{S}} v_{i} P_{i 0}(n) p_{00}^{(n)}=p_{00}^{(n)} \sum_{i \in \mathcal{S}} v_{i} P_{10}^{i}(n)
\end{aligned}
$$


where $P_{i 0}(n)=\mathbb{P}_{i}\left\{Z_{n}=0\right\}$ is a hitting probability to zero state of the process $\left\{Z_{n}\right\}$ without immigration and generated by PGF $F(s)$. Since this probability is equal to $F_{n}(0)$, one can see $\sigma^{n}=\mathcal{P}_{n}(0) \mathcal{U}\left(F_{n}(0)\right)$ and hence

$$
\mathcal{U}\left(F_{n}(0)\right)=\frac{\sigma^{n}}{p_{00}^{(n)}}
$$

for any $n \in \mathbb{N}$.

Let's consider the case $A \neq 1$. Due to continuity of $\mathcal{U}(s)$, from equality (2.8) we receive

$$
\frac{\sigma^{n}}{p_{00}^{(n)}} \longrightarrow \mathcal{U}(q)<\infty, \quad \text { as } n \rightarrow \infty
$$

Here we considered that $F_{n}(0) \rightarrow q$. Now considering together the relations (2.1), (2.4) and (2.9), we can write the following theorem.

Theorem 2. If $A \neq 1$, then

$$
\sigma^{-n} p_{i j}^{(n)} \longrightarrow \frac{q^{i} v_{j}}{\sum_{k \in \mathcal{S}} q^{k} v_{k}} \text {, as } n \rightarrow \infty
$$

for all $i, j \in \mathcal{S}$, where $\sigma=G(q)$ and $v_{j}=\lim _{n \rightarrow \infty} p_{0 j}^{(n)} / p_{00}^{(n)}$.

Further we expand our discussion concerning the equation (2.5) investigating properties of its solution.

Theorem 3. Let $A \neq 1$. Then there is a unique (up to a multiplicative constant) solution $\mathcal{U}(s)$ of the equation (2.5) for $s \in[0 ; q)$ such that

$$
\mathcal{L}(t)=\mathcal{U}(q-t)
$$

is a slowly varying function as $t \downarrow 0$.

Proof. Let's propose that there is another solution $\widehat{\mathcal{U}}(s)$ of the equation $(2.5)$. Then owing to equality $(2.6)$ we write

$$
\frac{\mathcal{U}(s)}{\widehat{\mathcal{U}}(s)}=\frac{\mathcal{U}\left(F_{n}(s)\right)}{\widehat{\mathcal{U}}\left(F_{n}(s)\right)}
$$

By definition the solution $\widehat{\mathcal{U}}(s)$ as well as $\mathcal{U}(s)$ monotonically increases. Since $F_{n}(0) \uparrow q$ then for given each $s \in[0 ; q)$ always there is $k \in \mathbb{N}$ such that $F_{k}(0) \leqslant s<F_{k+1}(0)$. Hence from equality (2.11) we will receive the following relations:

$$
\frac{\mathcal{U}(s)}{\widehat{\mathcal{U}}(s)} \leqslant \frac{\mathcal{U}\left(F_{n+k+1}(0)\right)}{\widehat{\mathcal{U}}\left(F_{n+k}(0)\right)}=\frac{\mathcal{U}\left(F_{n+k+1}(0)\right)}{\widehat{\mathcal{U}}\left(F_{n+k+1}(0)\right)} \cdot \frac{\widehat{\mathcal{U}}\left(F_{n+k+1}(0)\right)}{\widehat{\mathcal{U}}\left(F_{n+k}(0)\right)} .
$$

But again according to equality (2.11)

$$
\frac{\mathcal{U}\left(F_{n}(0)\right)}{\widehat{\mathcal{U}}\left(F_{n}(0)\right)}=\frac{\mathcal{U}(0)}{\widehat{\mathcal{U}}(0)}=1
$$

Then using once again (2.6) and the formula $\mathcal{P}_{n+1}(s)=\mathcal{P}_{n}(s) \cdot G\left(F_{n}(s)\right)$, we have

$$
\frac{\mathcal{U}(s)}{\widehat{\mathcal{U}}(s)} \leqslant \frac{\widehat{\mathcal{U}}\left(F_{n+k+1}(0)\right)}{\widehat{\mathcal{U}}\left(F_{n+k}(0)\right)}=\frac{\sigma}{G\left(F_{n+k}(0)\right)} .
$$


Taking limit as

$$
\frac{\mathcal{U}(s)}{\widehat{\mathcal{U}}(s)} \leqslant 1
$$

because PGF $G(s)$ continuously. By the similar way it is possible to establish the converse inequality $\mathcal{U}(s) / \widehat{\mathcal{U}}(s) \geqslant 1$. The received conclusions say that the equation (2.5) has a unique solution for all $s \in[0 ; q)$.

Now following a method of Seneta [8] we put

$$
g(s)=G(q-s), \quad f(s)=q-F(q-s) .
$$

In the allowed designations the equation (2.5) becomes

$$
\sigma \cdot \mathcal{L}(s)=g(s) \cdot \mathcal{L}(f(s)), \quad \text { for } s \in[0 ; q) .
$$

We will be convinced the function $f(s) / s$ monotonically decreases on set of $0 \leqslant s<q$ taking here the maximum $\beta=\lim _{s \downarrow 0}[f(s) / s]$ and the minimum $f(q) / q=1-p_{0} / q$ accordingly, where as before $\beta=F^{\prime}(q)$. The function $\mathcal{L}(s)$ in form of (2.10) is also monotonically decreasing on this set and $\lim _{s \downarrow 0} g(s)=\sigma$. Then for any $\lambda \in[\beta ; 1]$ one gets

$$
\frac{\mathcal{L}(f(s))}{\mathcal{L}(s)}=\frac{\mathcal{L}\left(\frac{f(s)}{s} s\right)}{\mathcal{L}(s)} \geqslant \frac{\mathcal{L}(\beta s)}{\mathcal{L}(s)} \geqslant \frac{\mathcal{L}(\lambda s)}{\mathcal{L}(s)} \geqslant 1 .
$$

On the other hand according to (2.12),

$$
\frac{\mathcal{L}(f(s))}{\mathcal{L}(s)}=\frac{\sigma}{g(s)} \longrightarrow 1, \quad \text { as } \quad s \downarrow 0 .
$$

Hence,

$$
\lim _{s \downarrow 0} \frac{\mathcal{L}(\lambda s)}{\mathcal{L}(s)}=1,
$$

for any $\lambda \in[\beta ; 1]$. It is easy to be convinced that the last relation is valid for any $\lambda \in \mathbb{R}_{+}$, where $\mathbb{R}_{+}$is set of positive real numbers. So $\mathcal{L}(s)=\mathcal{U}(q-s)$ is a slowly varying function as $s \downarrow 0$.

The Theorem is proved.

In the critical case it has been proved by Pakes [5] that the sequence $\left\{n^{\lambda} \mathcal{P}_{n}(s)\right\}$ converges to the limiting PGF $\pi(s)$ uniformly for $0 \leqslant s \leqslant r<1$ which is a solution of the equation (2.5):

$$
\pi(s)=G(s) \pi(F(s))
$$

where as before $\lambda=\alpha / B$. It was supposed therein that the moments

$$
\sum_{j \in \mathcal{S}} p_{j} j^{2} \ln j \quad \text { and } \quad \sum_{j \in \mathcal{S}} h_{j} j \ln j
$$

are finite. An advantage of assertion of the Theorem 1 from aforementioned result of Pakes consists that in our case the invariant measure $\left\{v_{j}\right\}$ for GWPI and corresponding for it the equation (2.5) is established without any moment assumptions concerning distributions $\left\{p_{j}\right\}$ and $\left\{h_{j}\right\}$. In the final Section of the paper we investigate a speed of convergence

$$
n^{\lambda} \mathcal{P}_{n}(s) \longrightarrow \pi(s), \quad \text { as } n \rightarrow \infty,
$$

strengthening aforementioned result of Pakes. 


\section{A speed rate of convergence to invariant measures in critical situation}

Consider the critical GWPI with transition functions $p_{i j}^{(n)}=\mathbb{P}_{i}\left\{X_{n}=j\right\}$. Recall the appropriate PGF

$$
\mathcal{P}_{n}(s)=\sum_{j \in \mathcal{S}} p_{0 j}^{(n)} s^{j}=\prod_{k=0}^{n-1} G\left(F_{k}(s)\right),
$$

where $G(s)$ and $F(s)$ are PGF's of immigration stream law and the process offspring law accordingly. Provided that moments $G^{\prime \prime}(1)$ and $F^{I V}(1)$ are finite, Pakes [5] investigated a rate of convergence $\left\{n^{\lambda} \mathcal{P}_{n}(s)\right\}$ to the limiting PGF $\pi(s)=\sum_{j \in \mathcal{S}} \pi_{j} s^{j}$, which is being the solution of functional equation $\pi(s)=G(s) \cdot \pi(F(s))$. So the nonnegative numbers $\left\{\pi_{j}\right\}$ satisfy the relation

$$
\pi_{j}=\sum_{i \in \mathcal{S}} \pi_{i} p_{i j}^{(n)}
$$

In this section we improve aforementioned result of Pakes, holding to condition of the third order factorial moment of offspring PGF is finite.

Theorem 4. Let $A=1,2 B:=F^{\prime \prime}(1), \alpha:=G^{\prime}(1)$ and $\lambda=\alpha / B$. If $C:=F^{\prime \prime \prime}(1)<\infty$, then the sequence $\left\{n^{\lambda} \mathcal{P}_{n}(s)\right\}$ converges to $\pi(s)$ uniformly for $0 \leqslant s \leqslant r<1$, and besides

$$
n^{\lambda} \mathcal{P}_{n}(s)=\pi(s) \cdot\left(1+\Delta \cdot \frac{\ln b_{n}(s)}{b_{n}(s)}(1+o(1))\right), \quad \text { as } n \rightarrow \infty,
$$

where $\Delta:=\alpha\left(\frac{C}{6 B^{2}}-1\right)$ and

$$
b_{n}(s)=B n+\frac{1}{1-s}
$$

Proof. It follows from (3.1) that

$$
\begin{aligned}
n^{\lambda} \mathcal{P}_{n}(s) & =n^{\lambda} \prod_{k=0}^{n-1} G\left(F_{k}(s)\right)= \\
& =G(s) \prod_{k=1}^{n-1}\left(1+\frac{1}{k}\right)^{\lambda} G\left(F_{k}(s)\right)=G(s) \prod_{k=1}^{n-1} A_{k}(s),
\end{aligned}
$$

where $A_{k}(s)=\left(1+\frac{1}{k}\right)^{\lambda} G\left(F_{k}(s)\right)$. It is known that the infinite product $\prod_{k \in \mathbb{N}} A_{k}(s)$ and the series $\sum_{k \in \mathbb{N}}\left(A_{k}(s)-1\right)$ converge or diverge simultaneously. Therefore we investigate the last series. Using elementary expansion $(1+1 / k)^{\lambda}=1+\lambda / k+\varepsilon_{k}$, we have the following representation:

$$
A_{k}(s)-1=\frac{\lambda}{k}-\left(1-G\left(F_{k}(s)\right)\right)-\frac{\lambda}{k}\left(1-G\left(F_{k}(s)\right)\right)+\varepsilon_{k} G\left(F_{k}(s)\right),
$$

where $\varepsilon_{k}=O\left(1 / k^{2}\right)$. We write

$$
1-G(s)=\alpha \cdot(1-s)-\delta(s)(1-s),
$$


where $0 \leqslant \delta(s)=(1-s) G^{\prime \prime}(\theta) / 2$ and $s<\theta<1$; obviously that $\delta(s)=o(1)$ as $s \uparrow 1$. In turn we know that (see $[1$, p.74]):

$$
\left|1-F_{n}(s)\right| \leqslant 2\left(1-F_{n}(0)\right),
$$

and according to the Basic Lemma of the theory of critical processes $1-F_{n}(0) \sim 1 / B n$; see e.g. [1, p.19]. Hence from relations (3.4)-(3.6) we will easily be convinced that

$$
A_{k}(s)-1=O\left(\frac{1}{k^{2}}\right), \quad \text { as } k \rightarrow \infty,
$$

uniformly for $0 \leqslant s \leqslant r<1$. Last statement testifies to a uniform convergence of the series $\sum_{k \in \mathbb{N}}\left(A_{k}(s)-1\right)$ and hence the infinite product $G(s) \prod_{k \in \mathbb{N}} A_{k}(s)$. We denote this as

$$
\pi(s):=\lim _{n \rightarrow \infty} n^{\lambda} \mathcal{P}_{n}(s)=G(s) \prod_{k \in \mathbb{N}} A_{k}(s) .
$$

Now to the proof of relation (3.2), we will estimate the error term of difference $n^{\lambda} \mathcal{P}_{n}(s)-\pi(s)$. So using representation (3.3) and equality (3.7) we obtain

$$
\begin{aligned}
n^{\lambda} \mathcal{P}_{n}(s)-\pi(s) & =G(s) \cdot\left[\prod_{k=1}^{n-1} A_{k}(s)-\prod_{k=1}^{\infty} A_{k}(s)\right]= \\
& =G(s) \prod_{k=1}^{n-1} A_{k}(s) \cdot\left[1-\prod_{k=n}^{\infty} A_{k}(s)\right] .
\end{aligned}
$$

According to the positiveness property of PGF we see $A_{k}(s)>0$ for all $0 \leqslant s<1$. Then using the elementary inequality $\ln (1-x) \geqslant-x-x^{2} /(1-x)$ we write down the following equalities:

$$
\begin{aligned}
\ln \prod_{k \geqslant n} A_{k}(s) & =\sum_{k \geqslant n} \ln \left\{1-\left(1-A_{k}(s)\right)\right\}= \\
& =-\sum_{k \geqslant n}\left(1-A_{k}(s)\right)+\rho_{n}^{(1)}(s)=: \Sigma_{n}(s)+\rho_{n}^{(1)}(s),
\end{aligned}
$$

where

$$
\Sigma_{n}(s)=-\sum_{k \geqslant n}\left(1-A_{k}(s)\right)
$$

and

$$
\begin{aligned}
0 \geqslant \rho_{n}^{(1)}(s) & \geqslant-\sum_{k \geqslant n} \frac{\left[1-A_{k}(s)\right]^{2}}{A_{k}(s)}> \\
& >-\left(1-G\left(F_{n}(s)\right)\right) \sum_{k \geqslant n} \frac{1-A_{k}(s)}{A_{k}(s)}> \\
& >\frac{1-G\left(F_{n}(s)\right)}{G\left(F_{n}(s)\right)} \cdot \Sigma_{n}(s) .
\end{aligned}
$$

The monotone property of PGFs used in the last step.

Replacing $s$ by $F_{k}(s)$ it follows from (3.5) that

$$
1-G\left(F_{n}(s)\right)=\alpha\left(1-F_{n}(s)\right)+\delta\left(F_{n}(s)\right)\left(1-F_{n}(s)\right) .
$$


Owing to (3.6) and (3.10) $1-G\left(F_{n}(s)\right) \sim \lambda / n$ and hence the augend in $(3.9) \rho_{n}^{(1)}(s) \rightarrow 0$ always supposing the first term $\Sigma_{n}(s)$ has a finite limit as $n \rightarrow \infty$. In turn in our conditions and owing to $(3.6) \delta\left(F_{n}(s)\right)=O(1 / n)$. Therefore combining (3.4), (3.6) and (3.10) we will receive the following equality for first term in (3.9):

$$
\Sigma_{n}(s)=-\sum_{k \geqslant n}\left(\alpha\left(1-F_{k}(s)\right)-\frac{\lambda}{k}\right)+\sum_{k \geqslant n} O\left(\frac{1}{k^{2}}\right) .
$$

Further we use the following asymptotic expansion for the function $1-F_{n}(s)$ which holds in the conditions of our theorem:

$$
1-F_{n}(s)=\frac{1}{b_{n}(s)}+\widetilde{\Delta} \cdot \frac{\ln b_{n}(s)+K(s)}{\left(b_{n}(s)\right)^{2}}(1+o(1)),
$$

as $n \rightarrow \infty$, where $\widetilde{\Delta}=\frac{C}{6 B}-B$ and $K(s)$ is some bounded function depending on form of $F(s)$ and $b_{n}(s)$ is same as in the theorem statement. The formula (3.12) was established in the paper [16] and we have reduced it in a bit modified form. So $b_{n}(s)=O(n)$ as $n \rightarrow \infty$, considering the expansion (3.12), we rewrite the equality (3.11) in form of

$$
\Sigma_{n}(s)=-\alpha \widetilde{\Delta} \cdot \sum_{k \geqslant n} \chi(k)+\rho_{n}^{(2)}(s),
$$

where $\rho_{n}^{(2)}(s)=\sum_{k \geqslant n} O\left(1 / k^{2}\right)$ and

$$
\chi(k)=\frac{\ln b_{k}(s)}{\left(b_{k}(s)\right)^{2}} .
$$

One can see that the function $\chi(k)$ is positive and monotonically decreases with respect $k \in \mathbb{N}$ and for all $0 \leqslant s<1$.

We consider now an alternative function $\chi(t)$ for $t \in \mathbb{R}_{+}$. Obviously this function is positive, monotonically decreases and also is continuous. Moreover

$$
\int \chi(t) d t=\mathrm{X}(t)+\text { const }
$$

where

$$
\mathrm{X}(t)=-\frac{1}{B}\left(\frac{\ln b_{t}(s)}{b_{t}(s)}+\frac{1}{b_{t}(s)}\right),
$$

for $0 \leqslant s<1$ and $\mathrm{X}(t) \rightarrow 0$ as $t \rightarrow \infty$. Therefore due to the Mc'Loren-Cauchy test (see [18, pp. 283-284]) the following inequalities hold:

$$
\frac{1}{B} \frac{\ln b_{n}(s)}{b_{n}(s)}+\frac{1}{b_{n}(s)} \leqslant \sum_{k \geqslant n} \chi(k) \leqslant \frac{1}{B} \frac{\ln b_{n-1}(s)}{b_{n-1}(s)}+\frac{1}{b_{n-1}(s)} .
$$

By means of the last inequalities and considering that $b_{n}(s)=O(n)$ as $n \rightarrow \infty$ we write estimation

$$
\left|\sum_{k \geqslant n} \chi(k)-\frac{1}{B} \frac{\ln b_{n}(s)}{b_{n}(s)}\right|=O\left(\frac{1}{n}\right), \quad \text { as } n \rightarrow \infty,
$$

for $0 \leqslant s<1$. 
Almost obviously that $\rho_{n}^{(2)}(s)=\sum_{k \geqslant n} O\left(1 / k^{2}\right)=O(1 / n)$. Considering it and exploiting $(3.14)$ in (3.13) we will obtain

$$
\Sigma_{n}(s)=-\Delta \cdot \frac{\ln b_{n}(s)}{b_{n}(s)}+O\left(\frac{1}{n}\right), \quad \text { as } n \rightarrow \infty,
$$

where $\Delta=\alpha\left(\frac{C}{6 B^{2}}-1\right)$.

Return now to the equality (3.9). Due to (3.15) and the fact $1-G\left(F_{n}(s)\right) \sim \lambda / n$ arises $\rho_{n}^{(1)}(s)=O\left(\ln n / n^{2}\right)$. Then from (3.9) and (3.15) we conclude

$$
\prod_{k \geqslant n} A_{k}(s)=\exp \left\{-\Delta \cdot \frac{\ln b_{n}(s)}{b_{n}(s)}(1+o(1))\right\}, \quad \text { as } n \rightarrow \infty .
$$

Finally using last expansion in (3.8) with combination of the formula $1-e^{-x} \sim x, x \rightarrow 0$, we complete the theorem proof.

From Theorem 4 we receive the following

Corollary 1. In conditions of Theorem 4 the following assertion is valid

$$
n^{\lambda} p_{00}^{(n)}=\pi_{0}\left(1+\frac{\Delta}{B} \cdot \frac{\ln n}{n}+o\left(\frac{\ln n}{n}\right)\right), \quad \text { as } n \rightarrow \infty,
$$

where $\Delta$ is defined in Theorem 4 .

The following theorem generalizes the previous one.

Theorem 5. Let conditions of the Theorem 4 are satisfied. Then the sequence $\left\{n^{\lambda} \mathcal{P}_{n}^{(i)}(s)\right\}$ converges to the limiting function $\pi(s)$ uniformly on the set of $0 \leqslant s \leqslant r<1$, and

$$
n^{\lambda} \mathcal{P}_{n}^{(i)}(s)=\pi(s)\left(\delta_{n}^{(i)}(s)+\Delta_{n}^{(i)}(s) \cdot \frac{\ln b_{n}(s)}{b_{n}(s)}(1+o(1))\right)
$$

as $n \rightarrow \infty$, where $\Delta_{n}^{(i)}(s)=\Delta \cdot \delta_{n}^{(i)}(s)$ and

$$
\delta_{n}^{(i)}(s)=1-\frac{i}{b_{n}(s)},
$$

and expressions $\Delta$ and $b_{n}(s)$ are defined in Theorem 4.

Proof. Since $F_{n}(s) \leqslant 1$ and $F_{n}(s) \uparrow 1$ as $n \rightarrow \infty$, it follows from (1.2) and Theorem 4 that $\left\{n^{\lambda} \mathcal{P}_{n}^{(i)}(s)\right\}$ converges uniformly to $\pi(s)$ for $0 \leqslant s \leqslant r<1$. Write

$$
n^{\lambda} \mathcal{P}_{n}^{(i)}(s)=\left(F_{n}(s)\right)^{i} n^{\lambda} \mathcal{P}_{n}(s) .
$$

It is obvious for fixed $i$ and at large values of number $n$

$$
\left(F_{n}(s)\right)^{i}=1-i\left(1-F_{n}(s)\right)(1+o(1)) .
$$

From here and using (3.12) follows

$$
\left(F_{n}(s)\right)^{i}=1-\frac{i}{b_{n}(s)}(1+o(1)), \quad \text { as } n \rightarrow \infty .
$$


Now the theorem statement follows from equalities (3.17) and (3.18), with application of the statements (3.2) and (3.12).

Corollary 2. In conditions of Theorem 5 the following assertion is valid:

$$
n^{\lambda} p_{i j}^{(n)}=\pi_{j}\left(\delta_{n}^{(i)}+\Delta \delta_{n}^{(i)} \cdot \frac{\ln B n}{B n}(1+o(1))\right), \quad \text { as } n \rightarrow \infty,
$$

for all $i, j \in \mathcal{S}$, where $\delta_{n}^{(i)}=1-i / B n$.

\section{References}

[1] K.B.Athreya, P.E.Ney, Branching processes, Springer, New York, 1972.

[2] C.R.Heathcote, Corrections and comments on the paper "A branching process allowing immigration", Journal of the Royal Statistical Society, B-28(1966), 213-217.

[3] C.R.Heathcote, A branching process allowing immigration, Journal of the Royal Statistical Society, B-27(1965), 138-143.

[4] A.G.Pakes, Limit for the simple branching process allowing immigration, I. The case of finite offspring mean, Advances in Applied Probability, 11(1979), 31-62.

[5] A.G.Pakes, Futher results on the critical Galton-Watson process with immigration, Journal of Australian Mathematical Soceity, 13(1972), no. 3, 277-290.

[6] A.G.Pakes, On the critical Galton-Watson process with immigration, Journal of Australian Mathematical Soceity, 12(1971), 476-482.

[7] A.G.Pakes, Branching processes with immigration, Journal in Applied Probabiliry, 8 (1971), no. $1,32-42$.

[8] E.Seneta, On invariant measures for simple branching process, Journal in Applied Probabiliry, 8(1971), 43-51.

[9] E.Seneta, An explicit-limit theorem for the critical Galton-Watson process with immigration, Journal of the Royal Statistical Society, B-32(1970), no. 1, 149-152.

[10] E.Seneta, Functional equations and the Galton-Watson process, Advances in Applied Probability, 1(1969), 1-42.

[11] E.Seneta, The stationary distribution of a branching process allowing immigration: A remark on the critical case, Journal of the Royal Statistical Society, B-30(1968), no. 1, $176-179$.

[12] Y.Uchimura, K.Saitô, Asymptotic behavior of the Bernoulli type Galton-Watson branching process with immigration, Random Operators and Stochastic Equations, 23(2015), no.1, $1-10$.

[13] Y.Uchimura, K.Saitô, Limiting distributions of Galton-Watson branching processes with immigration, Communications on Stochastic Analysis, 6(2012), no. 2, 281-295. 
[14] Y.Uchimura, K.Saitô, Stationary distributions of the Bernoulli type Galton-Watson branching process with immigration, Communications on Stochastic Analysis, 5(2011), no. 3, $457-480$.

[15] A.M.Zubkov, Life-Periods of a Branching Process with Immigration, Theory Probab. Appl., 17(1972), no. 1, 179-188.

[16] S.V.Nagaev, R.Myhammedzhanova, Some limit theorems of the theory of branching random processes, Limit theorems and statistical inference, Fan, Tashkent, 1966, 90-112 (in Russian).

[17] B.A.Sevastyanov, Branching processes, Nauka, Moscow, 1971 (in Russian).

[18] G.M.Fikhtengolts, Course of differential and integral calculus, V. 2, Nauka, Moscow, 1970 (in Russian).

\section{Об асимптотическом поведении состояний ветвящихся процессов Гальтона-Ватсона с иммиграцией}

Азам А. Имомов

В работе рассматривается ветвящийся процесс с иммиграчией дискретного времени. Исследуются предельные свойства переходных вероятностей и их сходимость к инвариантным мерам. В критическом случае определяется скорость этой сходимости.

Ключевые слова: ветвящийся процесс, иммиграция, переходные вероятности, инвариантные меры, скорость сходимости к инвариантным мерам. 